\title{
Algorithms for Computing the Capacity of Markov Channels with Side Information
}

\author{
Shailendra K. Singh \\ Department of $\cdot$ ECE \\ Indian Institute of Science \\ Bangalore, 560012, India \\ shailopal. ece.iisc.ernet.in
}

\section{INTRODUCTION}

Consider a finite state, irreducible Markov channel $\left\{S_{k}\right\}$. At time $k$ the channel output $Y_{k}$ depends only on the channel input $\boldsymbol{X}_{k}$ and the channel state $\&$. Let $U_{k}$ and $V_{k}$ he the CSIT (channel state information at transmitter) and the CSIR (CSI at reciever) respectively. In this paper we provide algorithms to compute the capacity of the Markov channel under various assumptions on CSIT and CSIR where the assumption $P\left(S_{k}=s \mid U_{1}^{k}\right)=P\left(S_{k} \mid U_{k}\right)$ in [1] is not necessarily satisfied. Our algorithms are based on the extension of ideas developed in [3] to the channels with feedback.

\section{DisCRETE CHANNEL}

In this section we further assume perfect CSIR i.e., $V_{n}=S_{n}$ and $U_{n}=g\left(S_{n-d+1}^{n}\right)$ where $g$ is a deterministic function of $S_{n-d+1}^{n} \stackrel{\mathrm{A}}{=}\left(S_{n-d+1}, \ldots, S_{n}\right)$. Under these assumptions, we show that the capacity achieving distribution of $\boldsymbol{X}_{\boldsymbol{n}}$ can he made a function of the conditional distribution $\rho_{n}$ of $\boldsymbol{S}_{n}$ giveu $U_{1}^{n}$ i.e, of

$$
\rho_{n}(s)=P\left(S_{n}=s \mid U_{1}^{n}\right) .
$$

$\rho_{n}$ can be computed recursively and $\left\{\rho_{n}\right\}$ is a regenerative process which converges in distribution. We also show that the capacity of the channel is

$$
\lim _{n \rightarrow \infty} \frac{1}{n} \sum_{k=1}^{n} \sup _{P\left(X_{k} \mid \rho_{k}\right)} \sum_{s} I\left(X_{k} ; Y_{k} \mid S_{k}=s, \rho_{k}\right) \rho_{k}(s)
$$

and this limit exists a.s. The sup in (2) is taken over all distributions on the input alphabet.

Theorem 1 With the above mentioned assumptions on CSIT and perfect CSIR, the channel cnpacity of the finite state Markov channel is

$$
C=\int_{\beta} \sup _{P(X \mid \rho)} \sum_{s} I(X ; Y \mid S=s, \rho) \rho(s) d \mu(\rho)
$$

where $\mu$ is the limiting distribution of $\rho_{n}$ and (2) converges a.s. to (3).

\section{AWGN CHANNEL WITH FADING}

In this section we consider a Markov fading channel with additive white Gaussian noise (AWGN) with mean zero and variance one. The $X_{n}$ satisfies long term average power constraint $E\left[X_{n}^{2}\right] \leq \bar{P}$. Here we assume that the receiver has the perfect knowledge of instantaneous SNR, i.e. $V_{n}=S_{n} E\left[\left|X_{n}\right|^{2} \mid U_{1}^{n}\right]$ and the CSIT may be imperfect i.e. $U_{n}=g\left(S_{n-d+1}^{n}, Z_{n}\right)$ where $g$ is a deterministic function and $\{Z$,$\} is an iid noise$ sequence. $A s$ in the previous section here we show that the OPA (Optimal Power Allocation Policy) can be made a function of the conditional distribution $\rho_{n}$, defined in (1). We show that

$$
\frac{1}{n} \sum_{i=1}^{n} \sum_{S_{i}=s} \frac{1}{2} \log \left(1+s \gamma\left(\rho_{i}\right)\right) \rho_{i}(s)
$$

\author{
Vinod Sharma \\ Department of ECE \\ Indian Institute of Science \\ Bangalore, 560012, India \\ vinodece. iisc.ernet. in
}

converges a.s, as $n \rightarrow \infty$, for any power allocation policy $\gamma(\cdot)$. This limit equals the capacity if $\gamma(\cdot)$, a function of $\rho$, is the OPA policy with respect to the limiting distribution $\boldsymbol{p}$ of $\boldsymbol{p} \boldsymbol{i}$.

Theorem 2 With the above assumptions on CSIT and CSIR, the channel cnpacity of the finite state Markov fading channel with AWGN noise and avemge power constrain $\bar{P}$ is

$$
C=\max _{\gamma(\cdot): \int_{\rho} \gamma(\rho) d \mu(\rho) \leq \bar{P}} \int_{\rho} \sum_{s} \frac{1}{2} \log (1+s \gamma(\rho)) \rho(s) d \mu(\rho)
$$

where $\mu$ is the limiting distribution of $\rho_{n}$ and (4) converges a.s. to (5) for the OPA policy.

Etom the Lagrange multiplier and the Kuhn-Tucker conditions [2] we get that the optimal power allocation policy $\gamma(\cdot)$ should satisfy

$$
\sum \frac{s}{1+s \gamma(\rho)} \rho(s) \leq \lambda
$$

and $\gamma(\cdot) \geq 0$, for all $p$ for which $\mu(\rho)>0$. Solving (6) for the minimum $\lambda$ such that the average power constraint $\int_{o} \gamma(\rho) d \mu(\rho) \leq \bar{P}$ is satisfied gives the optimal power allocation policy.

\section{EXAMPLES}

Consider an AWGN channel with the fade values $s_{n}=$ $1.0, s_{1}=0.5, s_{2}=0.01, s_{3}=0.001$ and let the average power constaiut $\bar{P}$ he $20 d b$. The capacity with perfect CSIT and CSIR is $\mathbf{1 . 8 1}$ hits. We will take a 1 bit CSIT ( $U_{k}=\mathbf{0}$ if $S_{k} \in\{.001,0.01\}$ else 1) and perfect CSIR and the delay in the feedback of the CSI to the transmitter $d$. Now the assumptions in [1] are violated. Then the capacity of this channel with $d=0$ is $C_{0}=\mathbf{1 . 7 8 6}$ bits and with $d=1$ is $C_{1}=1.699$ bits. Fig. 1 shows the convergence of (4) for $d=0$ and $d=1$.

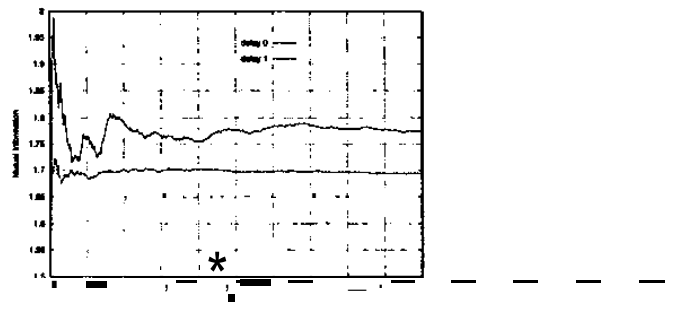

Figure 1: Convergence of Mutual Information to the capacity in Gaussian case

\section{REFERENCES}

11) G. Caire and S. Shamai, On the Capocity of Some Channels with Channel State Information, IEEE Transaction Information Theory, Vol. 42, 1996, pp. $868-886$.

[2] T. Cover and J. Thomas, Elements of Information Theory, Wiley, New York, 1991.

[3] V. Sharma, S. K. Singh Entropy rate and Mutual Information in the Regenerative Setup with Application to Morkoy Channels, Proc. IEEE Int. Symp. on Information Theory 2001, Washing. ton DC, USA June 2429, 2001. 